\title{
Innovación y competitividad en la agroindustria artesanal del queso crema de Chiapas
} \section{The innovation in artisan agro-industry of Chiapas'crem-chesse}

DOI: http://dx.doi.org/10.17981/econcuc.39.2.2018.02

Artículo de investigación. Fecha de recepción: 20/08/2018 Fecha de aceptación: 25/11/2018

Anastacio Espejel García

Universidad Autónoma Chapingo, Texcoco (México) anastacio.espejel@gmail.com

Carolina Illescas Marín

Universidad Autónoma Chapingo, Texcoco (México) illescasag@gmail.com

Arturo Hernández Montes

Universidad Autónoma Chapingo, Texcoco (México) hermora@prodigy.net.mx

Armando Santos Moreno

Universidad Autónoma Chapingo, Texcoco (México) armandosantoa_m@hotmail.com

Adán Ramírez García

Universidad Autónoma Chapingo, Obregón (México) gramirezg@taurus.chapingo.com

Para citar este artículo:

Espejel, A., Ilescas, C., Hernández, A., Santos, A. y Ramírez, A. (2018). Innovación y competitividad en la agroindustria artesanal del queso crema de Chiapas. Económicas CUC, 39(2). 25-38. DOI: http://dx.doi.org/10.17981/econcuc.39.2.2018.02

\section{Resumen}

El Queso Crema de Chiapas (QCC) está caracterizado por ser artesanal y típico del Sur de México. Los recursos locales y el saber hacer fortalecen su producción y conservan sus características. Estudios previos han caracterizado el producto, no así la adopción de innovaciones en la agroindustria. El objetivo de esta investigación fue medir la adopción de innovaciones y posición competitiva de las agroindustrias y proponer alternativas de mejora. Como estudio de caso, se seleccionaron once (11) queserías del municipio de Pijijiapan en Chiapas, debido a que son las de mayor colaboración y representativas; se seleccionaron empresas organizadas dentro de la marca colectiva y empresas no organizadas. Se clasificaron en cuatro estratos mediante un análisis jerárquico y la posición competitiva se obtuvo con el análisis de componentes principales (ACP). La adopción de innovaciones para el estrato 1 fue de $49 \%$ y de $18 \%$ para el 4 . Con respecto al ACP, dos componentes explican el $70 \%$ de la variabilidad $(\mathrm{KMO}=0.50 ; \mathrm{P}<0.05)$. La relación beneficio/costo fue de 1.14 para el estrato mejor posicionado y de 1.17 para el menor, si bien es mayor la relación para el estrato 4, en términos monetarios el 1 obtiene mayor utilidad. Se mostró mayor adopción de innovaciones en el estrato 1 quién tiene mayor utilidad y mejor posición competitiva, mientras que las menos competitivas presentan bajos e incluso nulos niveles de adopción en asistencia técnica y administración. La mejor posición competitiva se atribuye a implementación de innovaciones de mercado y administrativas adecuadas.

Palabras clave: innovación, agroindustria quesera, alimento tradicional

\section{Abstract}

The Chiapas'crem-chesse (CCC) is characterized by being traditional and typical of the South of Mexico. Local resources and know-how strengthen their production and preserve their characteristics. Previous studies have characterized the product, not so the adoption of innovations in agro-industry. The objective of this research was to measure the adoption of innovations and competitive position of agro-industries and propose alternatives of improvement. As a case study, eleven (11) cheese factories of the municipality of Pijijiapan in Chiapas were selected, because they are the most collaborative and representative; companies organized within the collective brand and non-organized companies were selected. They were classified into four strata through a hierarchical analysis and the competitive position was obtained with the principal components analysis (PCA). The adoption of innovations for stratum 1 was $49 \%$ and $18 \%$ for the 4 . With regard to the ACP, two components explain $70 \%$ of the variability $(\mathrm{KMO}=0.50, \mathrm{P}<0.05)$. The benefit / cost ratio was 1.14 for the best positioned stratum and 1.17 for the lowest, although the ratio is greater for stratum 4 , in monetary terms the 1 obtains greater utility. There was greater adoption of innovations in stratum 1, which has greater utility and better competitive position, while the less competitive one's present low and even null levels of adoption in technical assistance and administration. The best competitive position is attributed to the implementation of appropriate market and administrative innovations. Keywords: innovation, cheese agro-industry, traditional food 


\section{Introducción}

La agroindustria se concibe como el conjunto de operaciones posteriores a la cosecha relacionadas con el acondicionamiento, transformación, preservación y preparación de la producción agropecuaria para el consumidor intermedio o final; la cual resulta relevante en países en desarrollo ya que intensifica su crecimiento y el valor generado es tres veces más en comparación con la agricultura Food Agriculture Organization (FAO, 2013). La agroindustria de la producción de queso en México, en específico la del tipo artesanal, posee gran relevancia por constituir una fuente de empleo rural para diversos agentes que colaboran en la cadena agroalimentaria, con productos típicos, originales con calidad sensorial (Cervantes, Villegas y Cesin, 2016). Ejemplo de ello, es el Queso Crema de Chiapas(QCC), producto artesanal y típico derivado de un proceso histórico que da sustento a familias chiapanecas, que a través del tiempo, su proceso de producción y la forma de organizarse ha mejorado, incorporando a lo largo de la cadena diversas innovaciones, logrando posicionar al QCC de manera exitosa y competitiva (Villegas y Cervantes, 2011).

Sin embargo, la normativa mexicana vigente (NOM-243-SSA1-2010) prohíbe la elaboración de quesos con leche cruda, situación que pone en riesgo a la agroindustria del queso artesanal mexicano, demeritando a estos quesos genuinos, como productos no inocuos, con grandes riesgos para la salud, y desvalorizando la genuinidad y la tipicidad de estos productos (Villegas y Cervantes, 2011), impidiendo con ello la contribución al crecimiento sostenible del país (Chumaceiro, 2013). Una de las fortalezas de este grupo de queserías es la indicación geográfica que poseen (Marca colectiva), que plantea como propósito, el reconocimiento, distinción, valorización y preservación de este queso, producto de circulación local y regional, con creciente demanda de un grupo exclusivo de consumidores que busca productos de calidad con evocación de lo tradicional y genuino, respetando las reglas de uso bajo las cuales se produce este queso (Asociación de Procesadores de Queso Chiapas S.P.R. de R.L., 2013).

El entorno de las empresas ha cambiado, así como las demandas de los consumidores, debido a que son ellos, quienes impulsan a los otros eslabones y definen en gran medida la trayectoria tecnológica y la adopción de innovaciones (Abati e Imas, 2011; Zuñiga, 2011), la cual implica cambios basados en conocimientos específicos que agregan valor en diferentes aspectos en la cadena, producción, organización, proceso, producto y mercado; que no involucran grandes inversiones para su introducción, sino una estrategia para su desarrollo y continuidad contribuyendo principalmente en la disminución de costos y valorización del producto (Chen, 2006; Gellynck y Kühne, 2008). Es importante resaltar que se entiende por conocimiento lo que "se interpreta como capacidad de entender diferentes fenómenos humanos y tecnológicos desde una perspectiva formal y científica según los criterios disciplinarios para cada profesión" (Hernández y Chumaceiro, 2008, p. 78)

El éxito de las innovaciones se da por la adaptación y empleo de resultados de las actividades de investigación y desarrollo, dentro de un proceso interactivo entre los usuarios que permita una transferencia fácil y eficaz (Alburquerque, 2016; Méndez, 2006), siendo la proximidad elemento estratégico, por lo que los circuitos cortos de comercialización representan una alternativa eficiente para comercializar estos productos (Parker, 2005).

La aceptación o el rechazo de las nuevas tecnologías por parte de los consumidores, es el resultado de un proceso complejo de toma de decisiones que implica una evaluación de los riesgos/beneficios percibidos, asociados con la nueva tecnología y con características propias del producto (Henson, 1995). En México, existe de manera paralela la oferta de productos artesanales valorizados 
mínimamente por el consumidor y un mercado no identificado que, podría ser el nicho adecuado de estos productos. Para lograr esta articulación de manera equitativa es necesario realizar estudios de la agroindustria artesanal en términos de adopción de innovaciones, a partir de ello, este trabajo de investigación tiene el objetivo de analizar el nivel de adopción de innovaciones y su implicación en la posición competitiva de las empresas agroindustriales productoras de queso y con ello, proponer alternativas de mejora.

\section{Metodología}

La metodología esta basada en el análisis de las empresas queseras de la zona seleccionada mediante entrevistas suministraron la información, la cual se analizó y describió, así como tambien se hizo un análisis estadístico, razón por la cual el estudio fue del tipo mixto.

\section{Área y objeto de estudio}

El estudio fue realizado en la región de la Costa de Chiapas, en el municipio de Pijijiapan, donde se recopilaron datos de once (11) agroindustrias de queso crema a manera de estudio de caso; seleccionadas con base a su mayor propensión a colaborar y a la diversidad de tamaños de este tipo de empresas; adicionalmente se consideró que un grupo de ellas pertenecieran a la Marca Colectiva y otro grupo no.

\section{Análisis de innovación}

Se diseñó una encuesta sobre la dinámica de innovación integrada por cinco categorías: 1) producción, 2) manejo de residuos, 3) administración, 4) mercado, 5) asesoría técnica; considerando un total de 26 innovaciones (Producción: análisis fisicoquímico a leche, análisis sanitario a leche, análisis composicional a leche; análisis sanitario a queso, análisis composicional a queso, conocimiento y adopción de buenas prácticas de manufactura, estandarización del proceso, aplicación de nuevos ingredientes, empacado al vacío, uso de etiqueta, uso de marca registrada; manejo de residuos (lactosuero): directo al drenaje, alimento para animales, biogás, elaboración subproductos; administración: lineal, funcional, registros manuales, registros electrónicos; mercado: conoce las necesidades del cliente, conocimiento de la competencia, conocimiento de los precios en el mercado, análisis de nuevos canales de comercialización, promoción del producto; asesoría técnica: general, especializada de un programa, especializada pagada por el productor) que un buen productor quesero podría efectuar.

A partir de dicha encuesta se le preguntó a los productores queseros sobre datos generales, productivos y de adopción de las innovaciones. Los datos obtenidos fueron sistematizados en una base de datos empleando el software Microsoft Excel ${ }^{\circledR}$, con el posterior cálculo del índice de adopción de innovaciones (INAI) propuesto por Espejel, Barrera y Cuevas (2016), donde se asignó a las variables el valor de 1 si la empresa quisera introducir la innovación (ecuación 1):

$$
I N A I=\sum_{i=1}^{j} \begin{aligned}
& \begin{array}{l}
\text { Innovaciones realizadas } \\
\text { por el iproductor } i
\end{array} \\
& \begin{array}{l}
\text { Máximo número } \\
\text { de innovaciones por } \\
\text { los productores } j
\end{array}
\end{aligned} * 100
$$

\section{Análisis económico}

El análisis económico de las queserías se realizó considerando todos los costos estimados relacionados con la producción de Queso Crema de Chiapas (materia prima, insumos, distribución, empaque), además de considerar los ingresos totales brutos obtenidos en la unidad de producción (cantidad de quesos producidos multiplicada por el precio promedio). Las ganancias económicas se determinaron mediante la metodología propuesta por Espejel et al., (2016). empleando la ecuación 2: 


$$
B=\sum_{i=1}^{i} \quad I T-\sum_{i=1}^{i} C T
$$

Donde:

$B=$ Beneficios o ganancias económicas de la actividad quesera

$I T=$ suma de los ingresos totales de la unidad productiva (UP) de 1 hasta $n$.

$C=$ Suma de los costos de producción incurridos en la UP de 1 hasta $n$.

\section{Análisis estadístico}

Los datos obtenidos de las encuestas fueron organizados en una base de datos en el paquete Microsoft Excel®, posteriormente se agruparon con base a un análisis de conglomerados jerárquicos donde se realizó un análisis de componentes principales para la reducción de variables e identificar las relacionadas con la posición competitiva con el programa XLSTAT Versión 2014.5.03.

\section{Resultados}

\section{Caracterización de la agroindustria}

La agroindustria del Queso Crema de Chiapas se caracteriza por estar integrada de productores con una escolaridad promedio de 9 años, equivalente al nivel secundaria, con 57 años de edad y 23 años de experiencia, con un proceso de producción diario que va de los 200 a los 4,500 litros (promedio 1,245 L.) y un rendimiento quesero promedio de $9.9 \mathrm{~L}$. Cuatro de los productores queseros entrevistados, pertenecen a la Asociación de Procesadores de Queso Crema de Chiapas S.P.R. de R.L. quienes poseen una marca colectiva, lo cual representa una ventaja competitiva para estas queserías por su mayor desarrollo tecnológico, identidad y tipicidad asociada e integración al mercado.
La producción de queso es una de las principales actividades económicas para las familias; sin embargo, el Queso Crema de Chiapas para el $64 \%$ de los productores encuestados, sólo representa un porcentaje de dicho ingreso, debido a que algunos productores elaboran otros tipos de quesos, como el quesillo, queso de sal y cotija con los que complementan su producción e ingresos y estos son elaborados en la mayoría de las queserías con el apoyo de mano de obra familiar, lo que representa un costo que en muchas ocasiones no es considerado por los productores.

\section{La proveeduría en las empresas queseras del QCC}

La obtención de materia prima (leche de vaca) para el abastecimiento de las queserías se da a través de ruteros o productores independientes locales, que diariamente entregan su leche a los establecimientos, variando el precio de esta, si se incluye o no el costo de transporte. El número de proveedores que abastecen a las queserías oscila entre 1 y 45 , implicando para algunas queserías una elevada heterogeneidad de su materia prima que bien manejada representa una oportunidad en términos de riqueza composicional y de flora bacteriana nativa.

El volumen procesado promedio es de 1,245 L. por día, fluctuando para cada quesería, debido a la capacidad de producción de estas (Figura 1), dicha leche es destinada en parte para la obtención de Queso Crema de Chiapas elaborado de manera artesanal. Por otra parte, el precio por litro de leche está influenciado por la estacionalidad variando desde $\$ 4.5$ (época de lluvias) hasta $\$ 6.00$ (época de estiaje), con un promedio anual de $\$ 5.44$ por litro. Las queserías con mayor volumen de leche procesada fijan el precio de la leche por pertenecer a la Asociación de Procesadores de Queso Crema de Chiapas. 


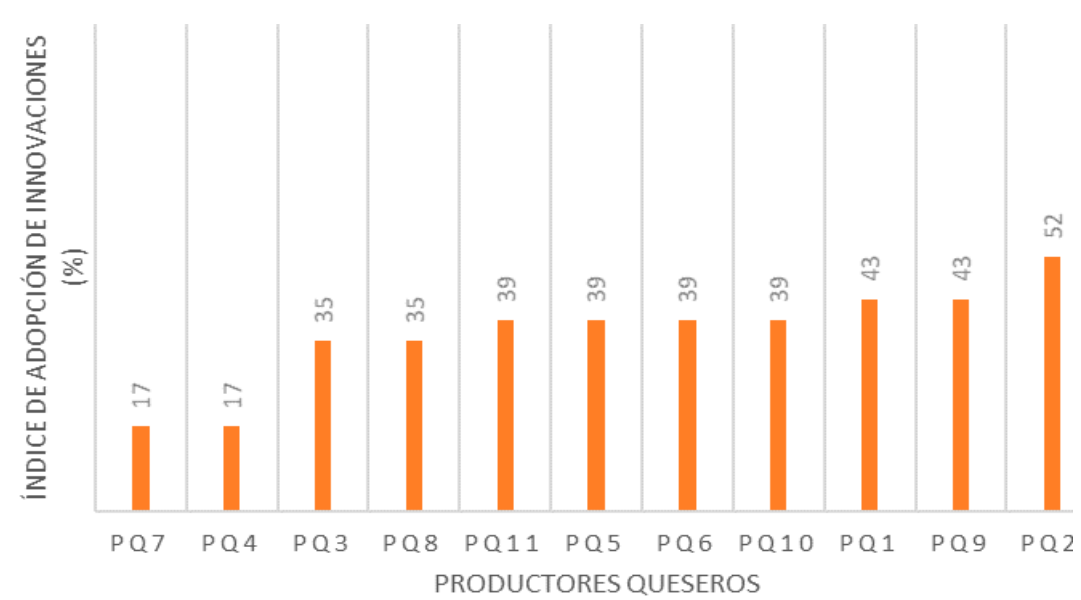

Figura 1. Índice general de adopción de innovaciones de productores de queso crema de Chiapas, del municipio de Pijijiapan, Chiapas.

Fuente: elaboración propia.

\section{Mercado}

Una de las características peculiares de las pequeñas empresas es el aprovechamiento de la proximidad geográfica con el mercado y que fortalecen con la venta local que realizan, la cual representa un soporte en la economía local y cada vez tiene más sentido a medida que se incrementan los consumidores (Demartini, Gaviglio y Pirani, 2017). Caso de ello son las pequeñas agroindustrias queseras del municipio de Pijijiapan, que realizan parte de la distribución y venta ahí mismo, lo que implica costos de transacción más bajos, lo cual fortalece la competitividad de las empresas. Mediante esta estrategia, Comercializan cerca del $25 \%$ de su producción total fijando diferentes precios de acuerdo con el canal de comercialización y la época del año; precio que oscila desde $\$ 55.00$ en temporada de lluvia hasta $\$ 96.00$ por Kg. de queso en temporada de seca.

Lo cual, según Chumaceiro y Hernández (2016) es dado por dos elementos: 1) el imperativo moral, que en momentos de globalización, se hace más exigible hacia el sector empresarial, y 2) las exigencias del correcto funcionamiento de los mercados. Randelli \& Rocchi (2017) por su parte, mencionan que la presión de los consumidores opera de ma- nera más eficiente a nivel local y puede apoyar la creación de nuevos mercados a partir de preferencias y mayor valorización de los productos, en este sentido los productores de QQC distribuyen una parte importante en el mercado local creciente, aunque, se han visto inmersos en un proceso de competencia local y regional que los ha obligado a diversificar sus mercados y con ello innovar en los mecanismos de distribución. En la actualidad, las empresas queseras, casi todas, poseen estrategias de comercialización que van desde la venta directa al consumidor en la propia quesería hasta la distribución en Centrales de Abastos que a su vez distribuyen a cremerías, teniendo como principales destinos la Ciudad de México; Tehuantepec, Oaxaca; Tapachula, Ocosingo y Comitán, Chiapas; Veracruz, Minatitlán y Coatzacoalcos en Veracruz.

Los mecanismos, por cierto, innovadores, por los que hacen llegar su producto a los consumidores ya cautivos en esos destinos, son: venta directa, envío por paquetería a través de autobuses, y por paquetería comercial. El volumen de queso semanal destinado a canales foráneos representa el $75 \%$ de su producción, esto contribuye a que se reconozca el QCC como un producto artesanal distintivo de esta región de Chiapas. 
El $81 \%$ de las queserías asegura tener un mercado fijo, estrategia construida con base a la fidelidad y reconocimiento de la calidad del producto por los clientes; se han abierto más canales influenciados por el turismo, los cuales adquieren el producto por primera vez y regresan a adquirir más. La exigencia de los clientes, en su mayoría, está relacionada con el peso exacto en cada unidad de queso, entrega oportuna, facturación para algunos clientes y quesos con etiqueta indicando la fecha de caducidad; dicha información de etiquetado, puede actuar como una guía conveniente para que los consumidores tomen decisiones rápidas de acuerdo a sus preferencias (Verbeke, 2005); innovación que ha sido introducida por el total de las queserías analizadas permitiendo mayor reconocimiento, diferenciación y aceptación del producto.

\section{Adopción de innovaciones en la agroindustria}

La innovación considerada como el momento en que un nuevo producto, servicio o proceso se introduce en el mercado eficientemente, a través de la interacción de actores e instituciones, forma parte esencial de la competitividad de las empresas como la publicidad. En este entendido, Núñez y Ravina (2017) manifiestan que los rankings de publicidad tienen una alta repercusión en los medios, haciendo notoria la marca y prestigio de las agencias, ya que sus resultados tienen gran capacidad para contener y simplificar las características de un sistema complejo.

Los productores de QCC poseen en promedio un área de procesamiento de $105 \mathrm{~m} 2$ y equipo básico para la producción de QCC; el $36 \%$ cuenta con tinas de acero inoxidable y descremadora, el $64 \%$ prensa de acero inoxidable, el $45 \%$ mesas de acero inoxidable y el $55 \%$ moldes de acero inoxidable. Complementario a estos equipos poseen tinas de plástico, artesas de madera, moldes de madera, prensas de madera y balanzas que, si bien no son sofisticados, son funcionales.
Cinco de las queserías que procesan más de $800 \mathrm{~L}$. de leche por día y cuentan con una planta de producción independiente, equipo básico de mediano grado de tecnificación que les permite obtener un producto con mayor nivel de inocuidad al emplear en su mayoría acero inoxidable.

El resto de las queserías, en su mayoría de pequeños productores, emplean equipo de plástico, acero, madera; el mantenimiento de este es mínimo, en parte por la baja sofisticación y en parte por la inversión que representa, lo cual lleva al mínimo los costos por este concepto; en caso extremo recurren a la sustitución de materiales como es el caso de los moldes de madera y de plástico. El grado de tecnificación en la elaboración de QCC es bajo, por el volumen de leche procesada y maquinaria y equipo empleado en el proceso, sin embargo, lo realmente importante es el saber hacer mediante el cual se elabora este producto. Algunas queserías, las mínimas, con mayor grado de tecnificación cuentan con un plan de mantenimiento preventivo para sus equipos e instalaciones.

El nivel de adopción de innovaciones es parte fundamental para la evaluación eficaz de los sistemas y su continuidad en el mercado, conforma una estrategia clave que permite identificar innovaciones con distinto nivel de madurez, funcionalidad y adaptación al sistema (Espejel, Ramírez y Mora, 2017). En este contexto, se identifica que los productores de Queso Crema de Chiapas, del municipio de Pijijiapan, son heterogéneos y no tienen un patrón específico en cuanto al volumen de procesamiento de leche y el nivel de adopción de innovaciones (Figura 1), donde se aprecia que los productores de QCC adoptan innovaciones en un rango del $17 \%$ al $52 \%$, por lo que la brecha tecnológica es aún elevada.

Los productores de QCC que poseen bajos índices de adopción de innovaciones son los que cuentan con bajos niveles de producción, con un espacio físico adaptado para el procesamiento artesanal, material y equipo de procesamiento en su mayoría de plástico y 
madera. En comparación con los productores con mayor adopción de innovaciones, quienes cuentan con un sistema más tecnificado que han ido adaptando a través del tiempo, por medio de capacitaciones, y demanda de los clientes, aunado a que procesan mayor volumen de leche y con ello obtienen mayores ingresos por la actividad; bajo este panorama, los productores más capacitados y mejor involucrados con mercados de mayor valor e instituciones de enseñanza e investigación y gubernamentales son los que muestran un mejor desempeño en la adopción de innovaciones y posición competitiva (Espejel et al., 2016).
El análisis de la adopción de innovaciones para las queserías de QCC, se calculó considerando cinco rubros con un total de 26 innovaciones que la agroindustria quesera podría estar adoptando; siendo el rubro de administración y de mercado los de mayor incidencia e impacto (Figura 2). El índice de adopción general promedio fue del $36 \%$, lo que sugiere que los productores de QCC aplican en promedio el 9.4\% de todas las innovaciones. Derivado de la estratificación de las queserías por medio del análisis de conglomerados jerárquicos (AHC), se observó que, en rubros como la

TABLA 1.

Adopción de innovaciones por estratos de la agroindustria del queso crema de Chiapas, de Pijijiapan, Chiapas (\%).

\begin{tabular}{lllll}
\hline Variables & Estrato 1 & Estrato 2 & Estrato 3 & Estrato 4 \\
\hline Asesoría técnica & 50 & 0 & 0 & 0 \\
Producción & 25 & 31 & 22 & 17 \\
Manejo de residuos & 25 & 25 & 25 & 25 \\
Administración & 70 & 65 & 60 & 0.0 \\
Mercado & 75 & 58 & 100 & 50 \\
Promedio & 49 & 38 & 41 & 18 \\
\hline
\end{tabular}

Fuente: elaboración propia.

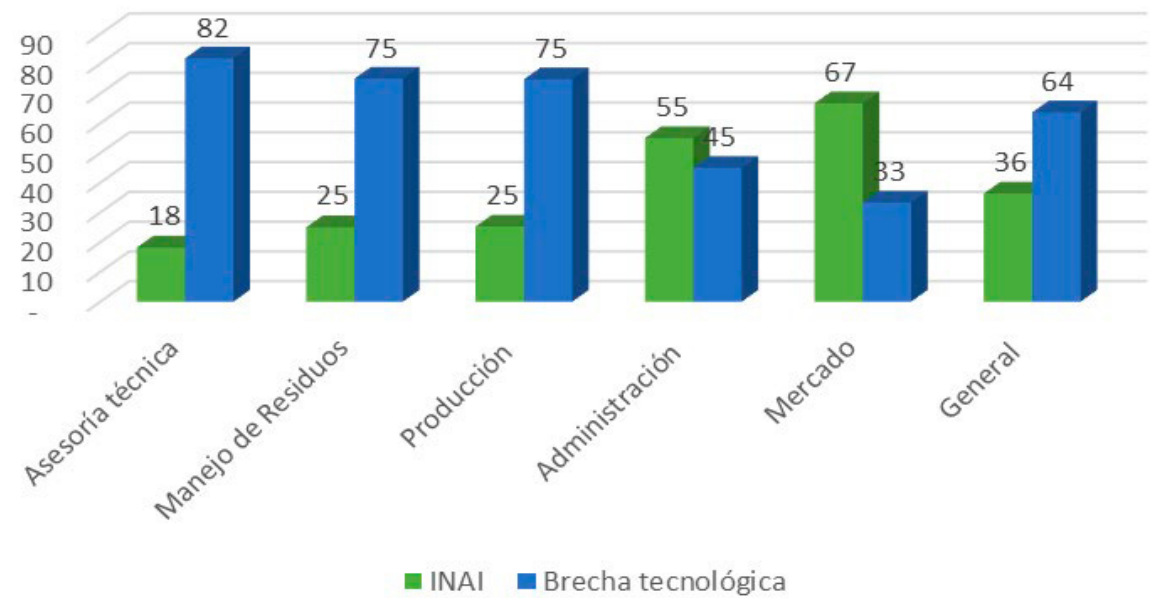

Figura 2. Adopción de innovaciones promedio en la agroindustria del queso crema de Chiapas. 
asesoría técnica, la adopción de innovaciones para tres estratos es nula, además, el estrato 4 (más débil) no considera a la administración como parte de la toma de decisiones y se carece de asesoría para llevar registros adecuados de su producción (Tabla 1).

Estudios realizados sobre el índice de adopción de innovaciones, obtuvieron, en el caso del quesillo de Reyes Etla, valores promedio del INAI global del $33 \%$ (Rodríguez, 2017), lo que asemeja al valor encontrado en el QCC. El papel del mercado y del consumidor han sido factores estratégicos para la continuidad y crecimiento de este sector agroindustrial, se evidencia que es uno de los de mayor relevancia, debido a que cada productor quesero opera en el mercado local y busca posicionarse en otros estados a partir de identificar las necesidades de sus clientes, así como el conocimiento de la competencia y el precio del producto en el mercado, esto ha permitido a las queserías competir e innovar constantemente en aspectos como el uso de etiqueta, promoción a través de redes sociales y liderazgo en precio, según el canal al que se destine el producto.

La integración de los productores queseros a la Asociación de procesadores de queso crema de Chiapas, S.P.R DE R.L, ha permitido a este grupo, acceder a asesoría técnica que se ve reflejada en el estrato 1 , en el cual el $80 \%$ han recibido capacitación especializada por parte de la
Universidad Autónoma Chapingo (UACh) y la Secretaria de Salud, a través de dicha Asociación, y el resto de los estratos no reciben ni contratan servicios similares. Situación contraria a la que presenta la agroindustria del queso añejo de Zacazonapan (Espejel et al., 2017), donde el rubro de capacitación impartida por la UACh y la UAEM obtuvo un INAI promedio del $38 \%$; distante al INAI promedio de $18 \%$ para la agroindustria del QCC. En este sentido, el papel de las universidades desempeña un rol estratégico en el desarrollo de capacidades hacia las organizaciones del sector social, desde la parte productiva hasta el mercado, propiciando una mayor propensión a la innovación (Arrieta, Rueda y Herrera, 2011).

Una de las necesidades en este tipo de agroindustria es el soporte para el manejo de los residuos, en este caso el manejo de lactosuero lo realizan apenas el $25 \%$ de las queserías y en los cuatro estratos la brecha tecnológica es del $75 \%$, esto implica que no han encontrado la importancia y el valor económicos que representaría el uso adecuado del lactosuero, pues actualmente se destina para alimentación de ganado y es recolectado por los propios proveedores de leche. Esto implica un proceso de concientización por parte de los productores sobre el deterioro del medioambiente, así como la vinculación con instituciones especialistas en estos temas y el desarrollo de capacidades.

TABLA 2

Estructura factorial del indice de adopción de innovaciones.

\begin{tabular}{llllll}
\hline & F1 & F2 & F3 & F4 & F5 \\
\hline Valor propio & 2.320 & 1.190 & 0.707 & 0.617 & 0.166 \\
Variabilidad (\%) & 46.40 & 23.81 & 14.15 & 12.33 & 3.31 \\
\% acumulado & 46.40 & 70.21 & 84.36 & 96.69 & 100.00 \\
\hline
\end{tabular}

Fuente: elaboración propia. 


\section{Posicionamiento competitivo de las agroindustrias de Queso Crema}

A partir de los resultados del índice de adopción de innovaciones y utilidad, se realizó un análisis de componentes principales para obtener la posición competitiva de las empresas agroindustriales. El diagrama de correlaciones que muestra la posición de las empresas queseras y el comportamiento de las variables de innovación y la utilidad se muestra en la figura 3. Dos factores explican el $70 \%(\mathrm{KMO}=0.50 ; \mathrm{P}<0.05)$ de la variabilidad total en las queserías artesanales de Queso Crema de Chiapas (Tabla 2).

Las queserías se agruparon en (4) categorías de acuerdo con las variables innovación y utilidad; las más destacadas poseen buenos indicadores de adopción de innovación en producción y administración, y con ello mayores utilidades. El grupo dos si bien no tiene variables cercanas asociadas, su relación beneficio costo es mayor que las queserías del grupo anterior; el grupo tres se caracteriza por la adopción de innovaciones en la parte de mercado y su fuerte vinculación con las instituciones que brindan asistencia técnica y capacitación; y el grupo cuatro se encuentra en proceso de crecimiento, y prevalece la baja adopción de innovaciones que no se reflejan totalmente en mejoras en la utilidad (Figura 3).

Con el análisis de conglomerados jerárquicos (AHC) se obtiene la figura 4, donde se agrupan los productores con la finalidad de visualizar características similares, obteniendo cuatro (4) agrupaciones que permiten ubicar a las empresas queseras y pre-

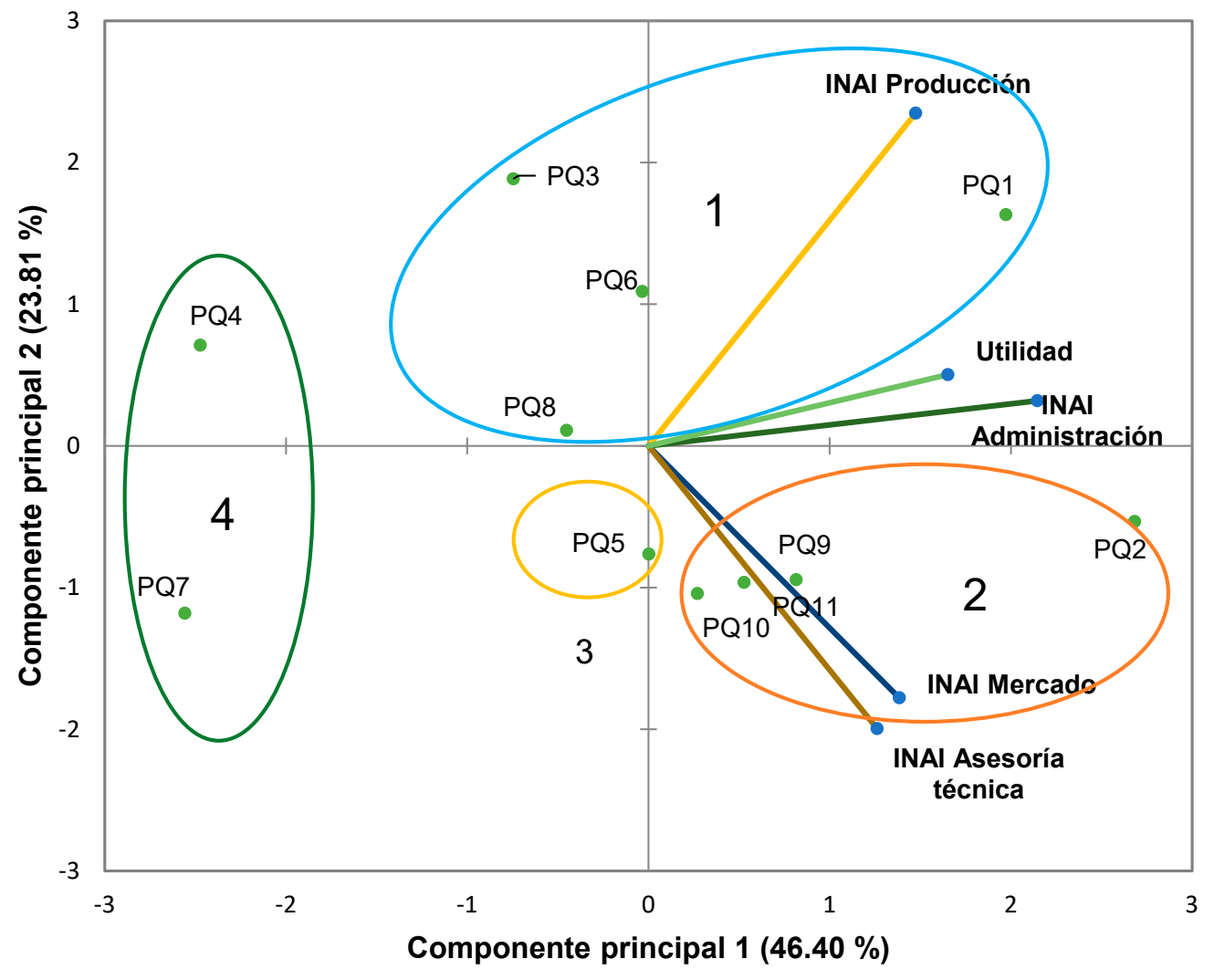

Figura 3. Cargas de los indices de adopción de innovaciones promedio y calificaciones de los productores queseros.

Fuente: elaboración propia. 


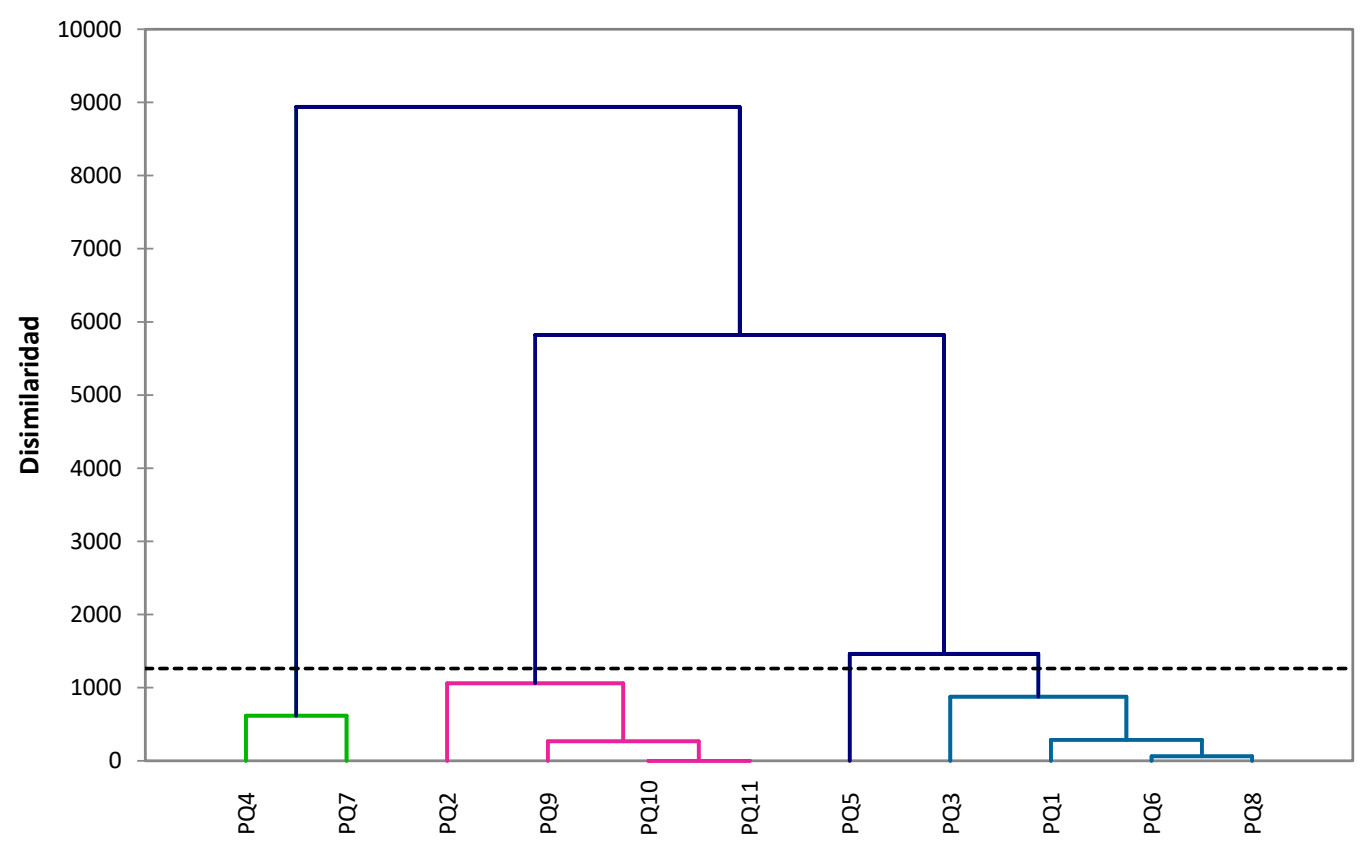

Figura 4. Dendograma de disimilaridad los productores de queso crema de Chiapas. Fuente: elaboración propia.

TABLA 3

Cargas factoriales de los componentes del índice de adopción de innovaciones y utilidad de las agroindustrias queseras de QCC.

\begin{tabular}{ccc}
\hline & F1 & F2 \\
\hline Producción & 0.6225 & $\mathbf{0 . 7 1 0 3}$ \\
Administración & $\mathbf{0 . 9 0 6 2}$ & 0.0964 \\
Mercado & 0.5843 & -0.5378 \\
Asesoría técnica & 0.5326 & -0.6034 \\
Utilidad & 0.6974 & 0.1521 \\
\hline
\end{tabular}

Fuente: elaboración propia.

cisar que aquellas con mayor utilidad son también las que adoptan innovaciones en producción y administración, índices que son los de mayor peso en los componentes 1 y 2 (Tabla 3). Así mismo, los productores con mayor INAI de mercado y asesoría técnica son quienes pertenecen a la Asociación de productores de QCC, lo que concuerda con Casas, De Fuentes, Torres y Vera-Cruz (2013). quienes mencionan que las organizaciones no innovan de manera aislada, sino en el contexto de un sistema.
Con respecto a la variable relación beneficio/costo, los cuatro estratos obtuvieron un valor mayor a 1.0 (Tabla 4), lo que significa que, por cada peso invertido, dicho peso fue recuperado más una ganancia extra para todas las queserías. La relación B/C promedio es de 1.15, mayor al obtenido para queso añejo de Zacazonapan, de 1.13 reportado por Espejel et al., (2017). Analizando a las agroindustrias por estrato, todas presentan utilidades, destacándose que la relación beneficio costo para el estrato 1 es mayor en compara- 
TABLA 4

Análisis económico de las agroindustrias del QCC por estrato

\begin{tabular}{ccccc}
\hline Variables & Estrato 1 & Estrato 2 & Estrato 3 & Estrato 4 \\
\hline Utilidad & 708,537 & 605,904 & 21,603 & 155,647 \\
Relación Beneficio-Costo & 1.14 & 1.17 & 1.05 & 1.17 \\
\hline
\end{tabular}

Fuente: elaboración propia.

ción con el estrato de menor adopción que es el 4; esto se atribuye a que los costos que se reportan no contemplan diversos rubros como el pago de impuestos, electricidad, entre otros. La composición de los costos totales que presentan el estrato 3 y 4 son mayores en la adquisición de materia prima, pues se encarece al comparar con proveedores y no estar integrados, así como elevado costo de insumos que son adquiridos en el municipio de Pijijiapan a menudeo, incrementando sus costos y reduciendo sus utilidades.

\section{Discusión}

Los alimentos tradicionales se consideran productos estratégicos al simbolizar pertenencia a un lugar, como parte de una sociedad y contribuyen a la valorización de recursos naturales y aspectos intangibles como cultura, valores y simbolismo, previniendo su desaparición (Guerrero, Claret, Verbeke, Enderli, Zakowska-Biemans, Vanhonacker y Hersleth, 2010; Muchnik, 2006). Ejemplo de ello, son los quesos genuinos de producción artesanal, producción limitada y en algunos casos con la finalidad de aprovechar los excedentes de leche en temporadas de lluvia (Grass, Sánchez, Altamirano, 2015).

La derrama económica generada es el sustento de familias, forma parte del arraigo cultural generacional que ha persistido durante décadas. Tal es el caso del queso crema de Chiapas, que ha logrado consolidarse a través de la demanda de los consumidores como un patrimonio cultural, avalado por la creación la indicación geográfica "Marca colectiva Chiapas Centenario" que tiene como función reglamentar, gestionar, promocionar y comercializar el Queso Crema de Chiapas en mercados locales, estatales, regionales y nacionales, además de establecer vínculos y convenios con diferentes actores sociales, como instituciones gubernamentales y universidades (Asociación de Procesadores de Queso Chiapas S.P.R. de R.L., 2013) lo que ha permitido para la agroindustria artesanal del QCC ir adoptando diferentes innovaciones, sin olvidar su origen tradicional.

La innovación considerada como el factor más importante de la competitividad y el éxito permite a los productores de queso conservar la calidad, mejorar su aspecto y con ello su posicionamiento en el mercado; sin embargo, es posible que los consumidores de cierto producto artesanal no acepten la innovación, la agroindustria entonces, podría innovar y aun así fallar en su objetivo con el consumidor (Guerrero, Claret, Verbeke, Sulmont y Hersleth, 2016), pero si los cambios introducidos son pequeños y pertinentes, es más probable que se cumpla el objetivo de la innovación y se acepte (Guerrero, Guàrdia, Xicola, Verbekeb, Vanhonacker, ZakowskaBiemans, Sajdakowska, Sulmont-Rossé, Issanchou, Contel, Scalvedi, Granlif y Hersleth, 2009; Guerrero et al., 2016). Ejemplo, son las empresas queseras de estudio, donde las innovaciones introducidas fueron el uso de etiqueta con marca registrada, para algunos productores permitió dar mayor relevancia al producto, además, de introducirse a nuevos canales de comercialización llegando al conocimiento de las necesidades del consumidor; dicha situación permite que la preferencia de los consumidores apoye a la creación de nuevos mercados de manera sostenible (Randelli y Rocchi, 2017). 
La vinculación de los pequeños productores con las instituciones es aún frágil en este tipo de sistemas, se encontró que las innovaciones con menor adopción fueron las de asesoría técnica; con respecto a la organización, solo $36 \%$ de los productores encuestados pertenecen a la Asociación de procesadores de Queso Crema de Chiapas S.P.R DE R.L., lo que conlleva a que los productores accedan a escasa o nula capacitación especializada por instituciones educativas o gubernamentales. Bajo este contexto, implica un escaso desarrollo de capacidades para los productores y limitado avance tecnológico (Sagastume, Obando y Martínez, 2006), lo que se traduce en un pequeño número de agroindustrias artesanales de QCC con capacidad innovadora y un bajo posicionamiento competitivo (Amaro-Rosales y De Gortari-Rabiela, 2016).

Para este grupo de empresas queseras la innovación es el elemento clave del posicionamiento competitivo, sin embargo dentro de las innovaciones, las relacionadas con el mercado y administrativas resultaron ser las más relevantes, al respecto es importante destacar que para productos artesanales como el analizado, la implementación de innovaciones es decisión de los productores lo que resulta en ocasiones una limitante si no reciben soporte institucional pertinente (Sagastume et al., 2006).

\section{Conclusiones e implicaciones}

La agroindustria artesanal del Queso Crema de Chiapas posee características que fortalecen su inserción en mercados locales, tales como la elevada disciplina en innovaciones relacionadas con el proceso de producción artesanal e inocuidad y el control adecuado del proceso administrativo reflejando utilidades positivas.

Pese a estar posicionadas de manera diferente, todas las queserías obtienen una relación beneficio/costo mayor a 1, sugieriendo lo rentable de la actividad y además demuestra una competitividad diferenciada por grupos de queserías en donde el mercado es quien impulsa a las empresas con mejor posición.
El grupo de queserías que están organizadas y que hacen uso de la marca colectiva poseen una elevada adopción de innovaciones en términos de estrategias de comercialización, seguramente por una mayor exigencia del consumidor, lo cual ha obligado a mejorar su producto y empaque en los últimos años.

La innovación incide de manera directa en la posición competitiva de las empresas queseras analizadas. Sin embargo, es importante promover innovaciones diferenciadas de acuerdo al nivel de desarrollo de las empresas, pues las más organizadas necesitan innovaciones relacionadas con el mercado, mientras que las menos desarrolladas o que procesan menor volumen deben atender innovaciones de proceso, administración y control de la materia prima.

\section{Agradecimientos}

Esta investigación fue financiada mediante el proyecto 17009-DTT, en su modalidad Desarrollo y Transferencia de Tecnología 2017, de la Dirección General de Investigación y Posgrado de la Universidad Autónoma Chapingo.

\section{Referencias}

Abati, G. e Imas, J. (2011). Innovar para satisfacer mejor al cliente. Harvard Deusto Marketing y Ventas, 104, 76-80.

Alburquerque, F. (2016). El enfoque del desarrollo económico territorial y la política. Enseñanzas para el cambio de modelo productivo. En, J. Noguera (Ed.), La visión territorial y sostenible del desarrollo local: una perspectiva multidisciplinar. Territorio, desarrollo, sistema productivo, Vol. 1, (47-66). Valencia: Universidad de Valencia.

Amaro-Rosales, M. y De Gortari-Rabiela, R. (2016). Innovación inclusiva en el sector agrícola mexicano: los productores de café en Veracruz. Economía Informa, 400, 86104. https://doi.org/10.1016/j. ecin.2016.09.006 
Arrieta, K., Rueda, Y. y Herrera, P. (2011). Estudio-diagnóstico de las capacidades tecnológicas para las Pymes cartageneras. AVANCES. Investigación en Ingeniería 8(2), 36-42.

Asociación de Procesadores de Queso Chiapas S.P.R. de R.L. (2013). Reglas de uso marca colectiva "Chiapas Centenario" queso crema de Chiapas. Recuperado de https://doc p l a $\mathrm{y}$ e $\mathrm{r}$. es/29387300-Chiapas-centenario-la-tradicion-no-se-vende-se-hereda-y-se-conservareglas-de-uso-marca-colectiva-queso-crema-de-chiapas.html

Casas, R., De Fuentes, C., Torres, A. y VeraCruz. A. (2013). Estrategias y gobernanza del sistema nacional de innovación mexicano: retos para un desarrollo incluyente. En, G. Dutrénit, y J. Sutz (Ed.), Sistemas de innovación para un desarrollo inclusivo. La experiencia Latinoamericana (35-64). Méxcio, D.F.: Foro Consultivo Científico y Tecnológico.

Cervantes, F., Villegas, A. y Cesín, A. (2016). Caracterización de la cadena agroindustrial del queso adobera de los Altos de Jalisco, México. En, Red del Queso Artesanal, ler Simposio de los quesos mexicanos genuinos. Chapingo, México.

Chen, K. (2006). Agri-food supply chain management: opportunities, issues, and guidelines. International conference on Livestock Services for Enhancing Rural Development. Beijing, China.

Chumaceiro, A. (2013). Políticas internacionales, nacionales y politicas públicas tributarias en Venezuela como promotoras de la responsabilidad social de las empresas. (Tesis doctoral). Universidad Dr. Rafael Belloso Chacín. Maracaibo

Chumaceiro, A. y Hernández, J. (2016). Países generadores de políticas públicas para el fomento de la cultura empresarial dirigida hacia la responsabilidad social. Revista Telos, 18(1). 103-120. Disponible en http:// ojs.urbe.edu/index.php/telos/article/ view/790
Demartini, E., Gaviglio, A. y Pirani, A. (2017). Farmers' motivation and perceived effects of participating in short food supply chains: evidence from a North Italian survey. Agric. Econ.-Checa, 63(5), 204-216. https:// doi.org/10.17221/323/2015-AGRICECON

Espejel, A., Barrera, A. y Cuevas, V. (2016). Dinámica de la innovación y ganancias económicas de la producción de leche en el Valle del Mezquital, Hidalgo. Revista Electrónica Nova Scientia, 8(2). 391-408.

Espejel, A., Ramírez, G. y Mora, E. (2017). Empleo e innovación en la producción de queso añejo en el municipio de Zacazonapan. En, Corposucre, Universidad, Ciencia, Innovación y Sociedad: desde la perspeciva laboral. (205-232). Colombia: Univesidad Simón Bolívar.

FAO. (2013). Agroindustrias para el Desarrollo. Roma: FAO.

Gellynck, X. y Kühne, B. (2008). Innovation and collaboration in traditional food chain networks. Journal on Chain and Network Science, 8(2). 121-129.

Grass, J., Sánchez, J. y Altamirano, J. (2015). Análisis de redes en la producción de tres quesos mexicanos genuinos. Estudios Sociales, 23(5). 185-212.

Guerrero, L., Claret, A., Verbeke, W., Enderli, G., Zakowska-Biemans, S., Vanhonacker, F., \& Hersleth, M. (2010). Perception of traditional food products in six European regions using free word association. Food Quality and Preference, 21(2), 225-233. https://doi.org/ $10.1016 / j$.foodqual.2009.06.003

Guerrero, L., Claret, A., Verbeke, W., Sulmont-Rossé, C., \& Hersleth, M. (2016). Chapter 5 - Innovation in Traditional Food Products: Does It Make Sense? In Ch. Galanakis, Innovation Strategies in the Food Industry. (77-89). Chania: Academic Press. $\quad$ https://doi.org/10.1016/C2015-000303-3

Guerrero, L., Guardia, M. D., Xicola, J., Verbeke, W., Vanhonacker, F., Zakowska-Biemans, S., Sajdakowska, M., Sulmont-Rossé, C., Issanchou, S., Contel, 
M., Scalvedi, M., Granli, B., \& Hersleth, M. (2009). Consumer-driven definition of traditional food products and innovation in traditional foods. A qualitative crosscultural study. Appetite, 52(2), 345-354. https://doi.org/10.1016/j.appet.2008.11.008

Gutiérrez, J. (2014). Costos sociales de transacción de la Ley de Restitución de Tierras en Colombia: Un país sin Reforma Agraria. Jurídicas CUC, 10(1), 157-196.

Henson, S. (1995). Demand-side constraints on the introduction of new food technologies: The case of food irradiation. Food Policy, 20(2), 111-127. https://doi.org/10.1016/03069192(95)00020-F

Hernández, J. y Chumaceiro, A. (2008). Sociedad del conocimiento y pertinencia social universitaria. CEISEP una propuesta para la UNERMB. Revista Venezolana de Ciencias Sociales. 12(1). 77-92.

Méndez, R. (2006). Del Distrito Industrial al Desarrollo Territorial: estrategias de innovación en ciudades intermedias. Desenvolvimento em Questão, 4(7). 9-46.

Muchnik, J. (2006). Identidad territorial y calidad de los alimentos: procesos de calificación y competencias de los consumidores. Agroalimentaria, 22. 89-98.

Núñez, E. y Ravina, R. (2017). Análisis del nivel de competitividad empresarial en el panorama publicitario colombiano fundamentada en el éxito creativo y la responsabilidad ética y jurídica. Jurídicas CUC, 13(1). 9-28. http://dx.doi.org/10.17981/juridcuc.13.1.2017.1

Parker, G. (2005). Sustainable food? Teikei, Co-operatives and food citizenship in Japan and the UK. Working Papers in Real Estate \& Planning 11/05. Available in http://centaur.reading.ac.uk/21289/1/1105.pdf

Randelli, F., \& Rocchi, B. (2017). Analysing the role of consumers within technological innovation systems: The case of alternative food networks. Environmental Innovation and Societal Transitions, 25, 94-106. https:// doi.org/10.1016/j.eist.2017.01.001
Rodríguez, D. (2017). Análisis de la red de valor y de innovación de la agroindustria del quesillo de Reyes, Etla, Oaxaca como una estrategia de fortalecimiento de la cadena. [Licenciatura]. Universidad Autónoma Chapingo, Chapingo, México.

Sagastume, N., Obando, M. y Martínez, M. (2006). Guía para la elaboración de estudios de adopción de tecnologías de manejo sostenible de suelos y agua. Tegucigalpa, Managua y San Salvador: PASOLAC, inter-cooperation, COSUDE.

Verbeke, W. (2005). Agriculture and the food industry in the information age. European. Review of Agricultural Economics, 32(3), 347-368.

Villegas, A. y Cervantes, F. (2011). La genuinidad y tipicidad en la revalorización de los quesos artesanales mexicanos. Estudios sociales, 19(38), 145-164.

Zuñiga, G. (2011). El desarrollo de cadenas de valor agroindustriales en Costa Rica, El Salvador y Nicaragua CEPAL (Ed.) El caso de estudio de la agroindustria láctea. México, D.F.: CEPL. Recuperado de https://repositorio.cepal.org/bitstrea m/ handle/11362/4911/1/S1100344_es.pdf

Anastacio Espejel García es profesor investigador del Posgrado en Ciencia y Tecnología Agroalimentaria. Universidad Autónoma Chapingo, México.

Carolina Illescas Marín es estudiante del Posgrado en Ciencia y Tecnología Agroalimentaria Universidad Autónoma Chapingo, México.

Arturo Hernández Montes es profesor investigador del Posgrado en Ciencia y Tecnología Agroalimentaria. Universidad Autónoma Chapingo, México.

Armando Santos Moreno es profesor investigador. Universidad Autónoma Chapingo, México.

Adán Ramírez García es profesor investigador. Universidad Autónoma Chapingo, Ciudad Obregón, México. 